\title{
Assessing the Pre-Analytical Stability of Small-Molecule Metabolites in Cerebrospinal Fluid Using Direct-Infusion Metabolomics
}

\author{
Hanneke A. Haijes ${ }^{1,2, *(\text { C }}$, Eline A.J. Willemse ${ }^{3}$, Johan Gerrits ${ }^{1}$, Wiesje M. van der Flier ${ }^{4,5}$, \\ Charlotte E. Teunissen ${ }^{3}$, Nanda M. Verhoeven-Duif ${ }^{1}$ and Judith J.M. Jans ${ }^{1, *}$ \\ 1 Section Metabolic Diagnostics, Department of Genetics, University Medical Center Utrecht, Utrecht \\ University, Lundlaan 6, 3584 EA Utrecht, The Netherlands; j.gerrits@umcutrecht.nl (J.G.); \\ N.Verhoeven@umcutrecht.nl (N.M.V.-D.) \\ 2 Section Metabolic Diseases, Department of Child Health, University Medical Center Utrecht, Utrecht \\ University, Lundlaan 6, 3584 EA Utrecht, The Netherlands \\ 3 Neurochemistry Laboratory and Biobank, Department of Clinical Chemistry, Amsterdam Neuroscience, \\ Amsterdam University Medical Center, Vrije Universiteit Amsterdam, De Boelelaan 1117, 1081 HV \\ Amsterdam, The Netherlands; e.willemse@amsterdamumc.nl (E.A.J.W.); \\ c.teunissen@amsterdamumc.nl (C.E.T.) \\ 4 Alzheimer Center and Department of Neurology, Amsterdam Neuroscience, Amsterdam University Medical \\ Center, Vrije Universiteit Amsterdam, De Boelelaan 1117, 1081 HV Amsterdam, The Netherlands; \\ wm.vdflier@amsterdamumc.nl \\ 5 Department of Epidemiology and Biostatistics, Amsterdam University Medical Center, Vrije Universiteit \\ Amsterdam, De Boelelaan 1117, 1081 HV Amsterdam, The Netherlands \\ * Correspondence: h.a.siepel-3@umcutrecht.nl (H.A.H.); J.J.M.Jans@umcutrecht.nl (J.J.M.J.)
}

Received: 28 August 2019; Accepted: 17 October 2019; Published: 18 October 2019

\begin{abstract}
Metabolomics studies aiming to find biomarkers frequently make use of historical or multicenter cohorts. These samples often have different pre-analytical conditions that potentially affect metabolite concentrations. We studied the effect of different storage conditions on the stability of small-molecule metabolites in cerebrospinal fluid to aid a reliable interpretation of metabolomics data. Three cerebrospinal fluid pools were prepared from surplus samples from the Amsterdam Dementia Cohort biobank. Aliquoted pools were exposed to different storage conditions to assess the temperature and freeze/thaw stability before final storage at $-80^{\circ} \mathrm{C}$ : storage up to four months at $-20^{\circ} \mathrm{C}$ and up to one week at either $5-8{ }^{\circ} \mathrm{C}$ or $18-22^{\circ} \mathrm{C}$ and exposure to up to seven freeze/thaw cycles. Direct-infusion high-resolution mass spectrometry was performed, resulting in the identification of $1852 \mathrm{~m} / \mathrm{z}$ peaks. To test the storage stability, principal component analyses, repeated measures analysis of variance, Kruskal-Wallis tests, and fold change analyses were performed, all demonstrating that small-molecule metabolites in the cerebrospinal fluid (CSF) are relatively unaffected by 1-3 freeze/thaw cycles, by storage at $-20^{\circ} \mathrm{C}$ up to two months, by storage at $5-8{ }^{\circ} \mathrm{C}$ for up to $72 \mathrm{~h}$, or by storage at $18-22^{\circ} \mathrm{C}$ for up to $8 \mathrm{~h}$. This suggests that these differences do not affect the interpretation of potential small-molecule biomarkers in multicenter or historical cohorts and implies that these cohorts are suitable for biomarker studies.
\end{abstract}

Keywords: biomarker stability; pre-analytical storage conditions; small-molecule metabolites; cerebrospinal fluid; metabolomics; direct-infusion mass spectrometry; DIMS; neurometabolic diagnostics

\section{Introduction}

The cerebrospinal fluid (CSF) is the closest possible read-out of all body fluids of metabolite concentrations in the brain, as it circulates in the subarachnoidal space and is in direct contact with the 
brain parenchyma, meninges, and spinal cord [1]. For this reason, CSF has been studied to identify biomarkers to aid diagnostics for a variety of neurological disorders, from neurodegenerative diseases and cerebral infections to inborn errors of metabolism.

Since studies aiming to identify biomarkers generally require large sample sizes, they frequently make use of historical or multicenter cohorts. However, the pre-analytical conditions in these cohorts, including the collection, processing, and storage of these samples, often vary [1]. These differences potentially affect the metabolite concentrations, resulting in bias [1]. Whereas studies on the effects of pre-analytical conditions on CSF often focus on protein stability [1], only a few studies have focused on the stability of small-molecule metabolites [2-4]. For example, one study found that the CSF concentrations of four amino acids (glutamic acid, glycine, aspartic acid, and taurine) were not affected by storage at $-20^{\circ} \mathrm{C}$ or $-80^{\circ} \mathrm{C}$, provided that the sample was deproteinized and neutralized before storage [2]. Another study demonstrated that storage for $72 \mathrm{~h}$ at room temperature, compared to immediate storage at $-70^{\circ} \mathrm{C}$, resulted in decreased levels of citrate and increased levels of lactate, glutamine, creatine, and creatinine, whereas the levels of myo-inositol, glucose, pyruvate, acetate, and alanine were unaffected [3]. Others showed that storage for 30 or $120 \mathrm{~min}$ at room temperature, compared to immediate storage at $-80{ }^{\circ} \mathrm{C}$, significantly increased the concentrations of $10 / 17$ amino acids measured in porcine CSF [4]. In addition, the majority of other measured small-molecule metabolites $(n=79)$ in this study [4] also demonstrated increased concentrations after $30 \mathrm{~min}$ and/or $120 \mathrm{~min}$ at room temperature, indicating that residual enzymatic activity in CSF after collection may lead to altered metabolite levels, possibly by the degradation of proteins [4], indicating that the storage conditions indeed affect the stability of small-molecule metabolites in CSF.

Although the studies performed to date give some indication of the potential effects of differences in pre-analytical storage conditions on the stability of small-molecule metabolites, only a limited number of metabolites were included in these studies, while targeted and untargeted metabolomics studies can potentially identify many more small-molecule metabolites. Thus, as targeted and untargeted metabolomics studies are more frequently performed for the identification of biomarkers in CSF, we studied the effect of different storage conditions on the stability of small-molecule metabolites in CSF to aid a reliable interpretation of metabolomics data.

\section{Results}

Untargeted metabolomics using direct-infusion high-resolution mass spectrometry resulted in a list of 1852 unique mass over charge $(\mathrm{m} / \mathrm{z})$ peaks, corresponding to 3806 unique annotations of metabolites that can occur endogenously, including isomers. This was in line with previous analyses [5,6]. In four out of 69 samples, direct infusion was hampered, resulting in the exclusion of the following samples: one of freeze/thaw cycle 1 (second CSF pool), one of freeze/thaw cycle 7 (first CSF pool), one of 5-8 ${ }^{\circ} \mathrm{C}$ for $168 \mathrm{~h}$, and one of $18-22{ }^{\circ} \mathrm{C}$ for $2 \mathrm{~h}$ (both in the third CSF pool). In total, 65/69 samples were used for statistical analyses. An overview of the different pre-analytical storage conditions assessed is given in Figure 1. 


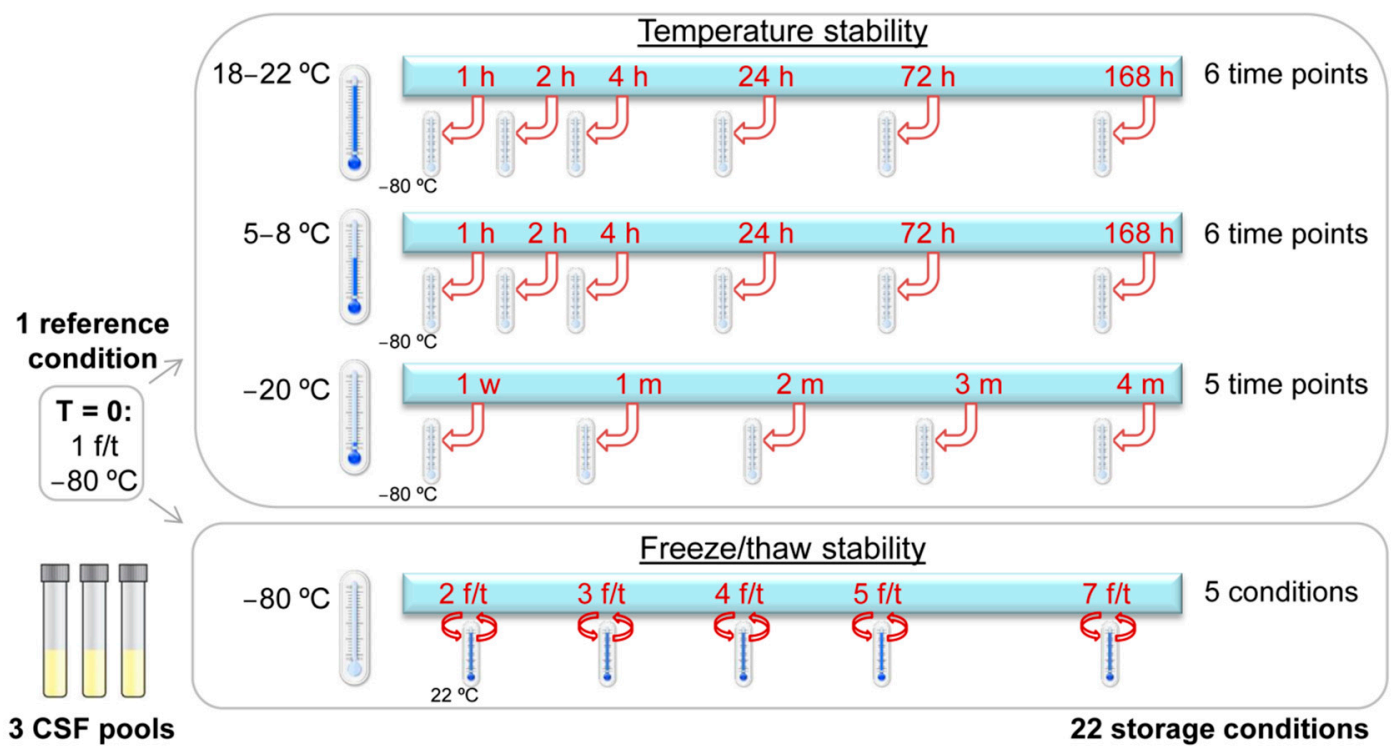

Figure 1. Overview of the different storage conditions analyzed to assess the temperature and freeze/thaw stability of small-molecule metabolites in cerebrospinal fluid. Three pools of cerebrospinal fluid were analyzed. For each pool, one reference sample and 22 samples for the different storage conditions were analyzed. Abbreviations: CSF: cerebrospinal fluid, f/t: freeze/thaw cycle, h: hours, m: months, w: week.

\subsection{Analysis of Variability.}

To assess which part of the identified variability is solely due to technical aspects of the analysis, the variability within the run was monitored by the addition of stable isotope-labeled compounds (sILC) to each sample during the sample preparation. The median coefficient of variation of the sILCs was 0.166 (95\% confidence interval: $0.127-1.565)$, in line with previous analyses [5] (Table 1).

As the sILCs were added during the sample preparation, the median absolute variation of the most extreme storage conditions was calculated as a reflection of the variation in the sample that can be solely attributed to analysis variability, and not to variability due to the different storage conditions tested. The median absolute variation of the sILCs was $0.384,0.072,0.171$, and 0.188 for the samples that experienced the most extreme storage conditions: freeze/thaw cycle 7 , storage at $-20^{\circ} \mathrm{C}$ for four months, storage at $5-8{ }^{\circ} \mathrm{C}$ for $168 \mathrm{~h}$, and storage at $18-22^{\circ} \mathrm{C}$ for $168 \mathrm{~h}$, respectively (Table 1 ). 
Table 1. Coefficient of variation of the analysis and median absolute variation of stable isotope-labeled compounds for the most extreme storage conditions.

\begin{tabular}{|c|c|c|c|c|c|}
\hline \multirow{2}{*}{$\begin{array}{l}\text { Stable Isotope-Labeled } \\
\text { Compound }\end{array}$} & \multirow[t]{2}{*}{$\mathrm{CV}$} & \multicolumn{4}{|c|}{ Median Absolute Variation } \\
\hline & & $\begin{array}{c}\text { Freeze/Thaw } \\
\text { Cycle } 7\end{array}$ & $\begin{array}{c}-20^{\circ} \mathrm{C}, \\
4 \text { Months }\end{array}$ & $\begin{array}{l}5-8^{\circ} \mathrm{C} \\
168 \mathrm{~h}\end{array}$ & $\begin{array}{c}18-22{ }^{\circ} \mathrm{C}, \\
168 \mathrm{~h}\end{array}$ \\
\hline${ }^{15} \mathrm{~N} ; 2-{ }^{13} \mathrm{C}$-glycine & 0.580 & 0.975 & 1.732 & 6.999 & 1.855 \\
\hline${ }^{2} \mathrm{H}_{4}$-alanine & 0.161 & 0.371 & 0.070 & 0.171 & 0.188 \\
\hline${ }^{2} \mathrm{H}_{3}$-leucine & 0.166 & 0.411 & 0.037 & 0.158 & 0.265 \\
\hline${ }^{2} \mathrm{H}_{3}$-methionine & 0.775 & 3.388 & 0.188 & 0.607 & 0.359 \\
\hline${ }^{13} \mathrm{C}_{6}$-phenylalanine & 0.149 & 0.374 & 0.050 & 0.142 & 0.204 \\
\hline${ }^{13} \mathrm{C}_{6}$-tyrosine & 0.150 & 0.349 & 0.043 & 0.098 & 0.206 \\
\hline${ }^{2} \mathrm{H}_{3}$-aspartate & 0.160 & 0.349 & 0.137 & 0.110 & 0.155 \\
\hline${ }^{2} \mathrm{H}_{3}$-glutamate & 0.126 & 0.247 & 0.099 & 0.110 & 0.074 \\
\hline${ }^{2} \mathrm{H}_{2}$-ornithine & 0.183 & 0.540 & 0.014 & 0.108 & 0.168 \\
\hline${ }^{2} \mathrm{H}_{2}$-citrulline & 0.134 & 0.231 & 0.070 & 0.131 & 0.038 \\
\hline${ }^{2} \mathrm{H}_{4},{ }^{13} \mathrm{C}$-arginine & 0.163 & 0.380 & 0.105 & 0.190 & 0.170 \\
\hline${ }^{2} \mathrm{H}_{8}$-valine & 0.155 & 0.377 & 0.042 & 0.124 & 0.222 \\
\hline${ }^{2} \mathrm{H}_{9}$-carnitine & 0.202 & 0.580 & 0.072 & 0.179 & 0.162 \\
\hline${ }^{2} \mathrm{H}_{3}$-acetylcarnitine & 1.058 & 432.707 & 689.785 & 0.567 & 0.999 \\
\hline${ }^{2} \mathrm{H}_{3}$-propionylcarnitine & 0.194 & 0.384 & 0.038 & 0.225 & 0.161 \\
\hline${ }^{2} \mathrm{H}_{3}$-butyrylcarnitine & 2.914 & 42.155 & 0.164 & 92.851 & 0.277 \\
\hline${ }^{2} \mathrm{H}_{9}$-isovalerylcarnitine & 0.217 & 0.519 & 0.103 & 0.215 & 0.173 \\
\hline 5th percentile & 0.127 & 0.244 & 0.033 & 0.106 & 0.067 \\
\hline Median & 0.166 & 0.384 & 0.072 & 0.171 & 0.188 \\
\hline 95th percentile & 1.565 & 120.266 & 139.343 & 24.170 & 1.170 \\
\hline
\end{tabular}

CV: coefficient of variation of all 65 samples, calculated by: standard deviation intensity/mean intensity. Median absolute variation for the most extreme storage condition was calculated by: (1) calculation of the fold change: intensity of the most extreme condition/intensity of the reference sample; (2) fold change - 1; (3) conversion to absolute numbers; (4) calculation of the median absolute variation: median of the absolute variation of the three CSF pools.

\subsection{Freeze/Thaw Stability}

To assess whether the metabolome changed due to multiple freeze/thaw cycles, principal component analysis was performed for the $1852 \mathrm{~m} / \mathrm{z}$ peaks as well as for the selection of $106 \mathrm{~m} / \mathrm{z}$ peaks corresponding to metabolites marked as important for neurometabolic diagnostics (Table 2). This did not reveal any clustering of the different freeze/thaw cycles (Figure S1), indicating that the metabolome as a whole did not change upon multiple freeze/thaw cycles. In addition, no clustering of the samples from the three different CSF pools was observed, suggesting that the three CSF pools had comparable metabolomes (Figure S1).

Repeated measures analysis of variance (ANOVA) assessing all $1852 \mathrm{~m} / \mathrm{z}$ peaks for all freeze/thaw cycles revealed no significant differences; nor did Kruskal-Wallis tests comparing freeze/thaw cycle 7 to freeze/thaw cycle 1 . When assessing the selection of $106 \mathrm{~m} / \mathrm{z}$ peaks corresponding to neurometabolic metabolites, neither the repeated measures ANOVA comparing all freeze/thaw cycles nor the Kruskal-Wallis tests comparing freeze/thaw cycle 7 to freeze/thaw cycle 1 revealed any significant differences, suggesting that the stability of individual $\mathrm{m} / \mathrm{z}$ peaks is unaffected by multiple freeze/thaw cycles. 
Table 2. $106 \mathrm{~m} / \mathrm{z}$ peaks corresponding to metabolites important for neurometabolic diagnostics in the cerebrospinal fluid.

\begin{tabular}{|c|c|c|c|}
\hline Amino Acids (24) & Neurotransmitters (21) & Purines, Pyrimidines (20) & Organic Acids (16) \\
\hline Alanine & 3-Methoxytyrosine/3-OMD/ & 5-Hydroxymethyluracil & N-Acetylaspartylglut. Acid \\
\hline Arginine & Methyldopa & Adenine & 2-Methylcitric acid \\
\hline Asparagine & 5-Hydroxyindoleacetic acid & Adenosine/Deoxyguanosine & 3-Hydroxybutyric acid \\
\hline Aspartic acid & 5-Hydroxytryptophan & AICAR & 3-Hydroxyisovaleric acid \\
\hline Cysteine & 5-Methyltetrahydrofolic acid & Deoxyadenosine & 4-Guanidinobutanoic acid \\
\hline Cystine & Dopamine & Deoxyinosine & Acetoacetic acid \\
\hline Glutamic acid & Dopamine 4-sulfate & Dihydrothymine & Cis-Aconitic acid \\
\hline Glutamine & Dopamine glucuronide & Dihydrouracil & Citric acid \\
\hline Glycine & Epinephrine & Guanosine & Fumaric acid \\
\hline Histidine & Epinephrine glucuronide & Hypoxanthine & Lactic acid/ \\
\hline Homoarginine & Homovanillic & Inosine & 3-OH-propionic acid \\
\hline Homocarnosine & acid/3-OH-phenyllactic acid & Orotic acid & Malic acid \\
\hline (Iso)leucine & Epinephrine sulfate & SAICAR & $\mathrm{N}$-Acetylaspartic acid \\
\hline Lysine & Gamma-aminobutyric acid & Succinyladenosine & Oxalacetic acid \\
\hline Methionine & Glutamic acid & Thymidine & Propionic acid \\
\hline Phenylalanine & L-Dopa/Dihydroxyphenylalan. & Thymine & Pyruvic acid \\
\hline Proline & Methoxyhydroxyphenylglycol & Uracil & Succinic acid/ \\
\hline Serine & $\mathrm{N}$-Acetylserotonin & Uric acid & \multirow{3}{*}{ Methylmalonic acid } \\
\hline Threonine & Norepinephrine & Uridine & \\
\hline $\begin{array}{l}\text { Tryptophan } \\
\text { Tyrosine }\end{array}$ & $\begin{array}{l}\text { Norepinephrine sulfate } \\
\text { Serotonin }\end{array}$ & Xanthine & \\
\hline Ornithine & Vanillactic acid & Other (1) & Methyldonor (2) \\
\hline $\begin{array}{l}\text { Taurine } \\
\text { Valine }\end{array}$ & Vanillylmandelic acid & Saccharopine & $\begin{array}{l}\text { s-Adenosylhomocysteine } \\
\text { s-Adenosylmethionine }\end{array}$ \\
\hline Creatine m. (5) & Vitamers (5) & Pterines (6) & Vitamin B6 vitamers (8) \\
\hline Creatine & Folic acid & 7,8-Dihydroneopterin & Pipecolic acid \\
\hline Creatinine & Thiamine & Dihydrobiopterin & Pyridoxal \\
\hline Guanidoacetic acid & Thiamine monophosphate & Neopterin & Pyridoxal 5'-phosphate \\
\hline Phosphocreatine & Thiamine pyrophosphate & Biopterin/Sepiapterin/Primapterin & Pyridoxamine \\
\hline \multirow[t]{4}{*}{ Phosphocreatinine } & Thiamine triphosphate & /6-Pyruvoil-tetrahydropterin & Pyridoxamine $5^{\prime}$-phosph. \\
\hline & & Tetrahydroneopterin & Pyridoxine \\
\hline & & & Pyridoxine $5^{\prime}$-phosphate \\
\hline & & & $\begin{array}{l}\text { Alpha-aminoadipic acid } \\
\text { delta-semialdehyde }\end{array}$ \\
\hline
\end{tabular}

To detect more subtle changes in metabolite stability, the freeze/thaw stability was further assessed by an analysis of fold changes (FC). After correction for the median absolute variation reflecting the analysis variability, 119 of the $1852 \mathrm{~m} / \mathrm{z}$ peaks were considered to be affected by the most extreme storage condition, of which six were neurometabolic metabolites (Table 3, Figure 2). There was no specific group of neurometabolic metabolites that was more affected than another group (Figure 2). The six metabolites that were possibly affected by multiple freeze/thaw cycles were visually assessed (Figure S2). Remarkably, adenine concentrations showed a decrease of more than $50 \%$ after more than four freeze/thaw cycles and glycine concentrations showed a more than five-fold increase after more than five freeze/thaw cycles (Figure S2). 
Table 3. Number of metabolites possibly affected by the most extreme storage condition, after correction for the analysis variability.

\begin{tabular}{|c|c|c|c|c|}
\hline & $\begin{array}{c}\text { Freeze/Thaw } \\
\text { Cycle } 7\end{array}$ & $\begin{array}{c}-20^{\circ} \mathrm{C}, \\
4 \text { Months }\end{array}$ & $\begin{array}{l}5-8^{\circ} \mathrm{C} \\
168 \mathrm{~h}\end{array}$ & $\begin{array}{c}18-22{ }^{\circ} \mathrm{C} \\
168 \mathrm{~h}\end{array}$ \\
\hline Median absolute variation sILC & 0.384 & 0.072 & 0.171 & 0.188 \\
\hline Range analysis variability & $0.616-1.384$ & $0.928-1.072$ & $0.829-1.171$ & $0.812-1.188$ \\
\hline \multicolumn{5}{|l|}{$1852 \mathrm{~m} / \mathrm{z}$ peaks } \\
\hline$m / z$ peaks with decreased intensities & 45 & 142 & 206 & 67 \\
\hline$m / z$ peaks with increased intensities & 74 & 134 & 213 & 54 \\
\hline \multicolumn{5}{|c|}{$106 \mathrm{~m} / \mathrm{z}$ peaks corresponding to neurometabolic metabolites } \\
\hline$m / z$ peaks with decreased intensities & 3 & 5 & 8 & 1 \\
\hline$m / z$ peaks with increased intensities & 3 & 6 & 15 & 5 \\
\hline
\end{tabular}

Groups of $m / z$ peaks are depicted in bold. The median absolute variation of the sILC, as demonstrated in Table 1 , reflects the variability in the sample that can be attributed to technical aspects of the analysis. The range of the analysis variability is calculated by a fold change of $1 \pm$ median absolute variation of the sILC. $\mathrm{m} / \mathrm{z}$ peaks were considered decreased when the upper limit of the $95 \%$ confidence interval of the $\mathrm{m} / \mathrm{z}$ peak was below the range of the analysis variability, and increased when the lower limit of $95 \%$ confidence interval of the $\mathrm{m} / \mathrm{z}$ peak was above the range of the analysis variability. Abbreviations: sILC: stable isotope-labeled compound.

\subsection{Temperature Stability}

For all three temperature stability series, i.e., storage at $-20{ }^{\circ} \mathrm{C}$, at $5-8{ }^{\circ} \mathrm{C}$ or at $18-22{ }^{\circ} \mathrm{C}$ for different periods of time (Figure 1), principal component analysis, performed to assess whether the metabolome changed due to the storage conditions, did not reveal any clustering of the different time points (Figure S1), suggesting that the metabolome as a whole was not affected by the different storage conditions. In addition, no clustering of CSF pools was observed, indicating that the metabolic compositions of the pools was comparable (Figure S1).

Repeated measures ANOVA assessing all time points and Kruskal-Wallis tests comparing the most extreme time points to the reference samples revealed no significant differences. For the selection of $106 \mathrm{~m} / \mathrm{z}$ peaks corresponding to neurometabolic metabolites, Kruskal-Wallis tests comparing the most extreme time points to the reference samples did not display any significant differences, and repeated measures ANOVA only disclosed a significant change in mass peak intensity for cis-aconitic acid when stored at $18-22{ }^{\circ} \mathrm{C}$, suggesting that the stability of individual $\mathrm{m} / \mathrm{z}$ peaks is unaffected by the different storage conditions, except for cis-aconitic acid when stored at $18-22^{\circ} \mathrm{C}$.

To detect more subtle changes, the temperature stability was further assessed by analysis of FCs. After correction for the median absolute variation reflecting the analysis variability, 276 of the $1852 \mathrm{~m} / \mathrm{z}$ peaks were considered to be affected by storage for four months at $-20{ }^{\circ} \mathrm{C}, 419 \mathrm{~m} / \mathrm{z}$ peaks were considered to be affected by storage for a week at $5-8{ }^{\circ} \mathrm{C}$ and $121 \mathrm{~m} / \mathrm{z}$ peaks were considered to be affected by storage for a week at $18-22{ }^{\circ} \mathrm{C}$ (Table 3). Of these peaks, 11,23 , and six ( 25 unique $\mathrm{m} / \mathrm{z}$ peaks) were among the $106 \mathrm{~m} / \mathrm{z}$ peaks corresponding to neurometabolic metabolites (Table 3 , Figure 2). No specific group of neurometabolic metabolites was more affected than another group (Figure 2). Metabolites that were possibly affected by prolonged storage at $-20^{\circ} \mathrm{C}, 5-8{ }^{\circ} \mathrm{C}$, and $18-22{ }^{\circ} \mathrm{C}$ were visually assessed (Figure S3-S5). 5-methyltetrahydrofolic acid concentrations were decreased after storage for more than two months at $-20^{\circ} \mathrm{C}$ and dopamine glucuronide concentrations were increased after storage for more than three months at $-20{ }^{\circ} \mathrm{C}$ (Figure S3). Prolonged storage at $5-8{ }^{\circ} \mathrm{C}$ resulted in a decrease in cis-aconitic acid, pyruvic acid, 2-methylcitric acid, and $\alpha$-aminoadipic acid delta-semialdehyde concentrations and an increase in orotic acid and neopterin concentrations (Figure S4). Lastly, prolonged storage at $18-22{ }^{\circ} \mathrm{C}$ resulted in a decrease in cis-aconitic acid, pyruvic acid, 2-methylcitric acid, $\alpha$-aminoadipic acid delta-semialdehyde and glutamine concentrations, and in an increase in adenine concentrations (Figure S5). 

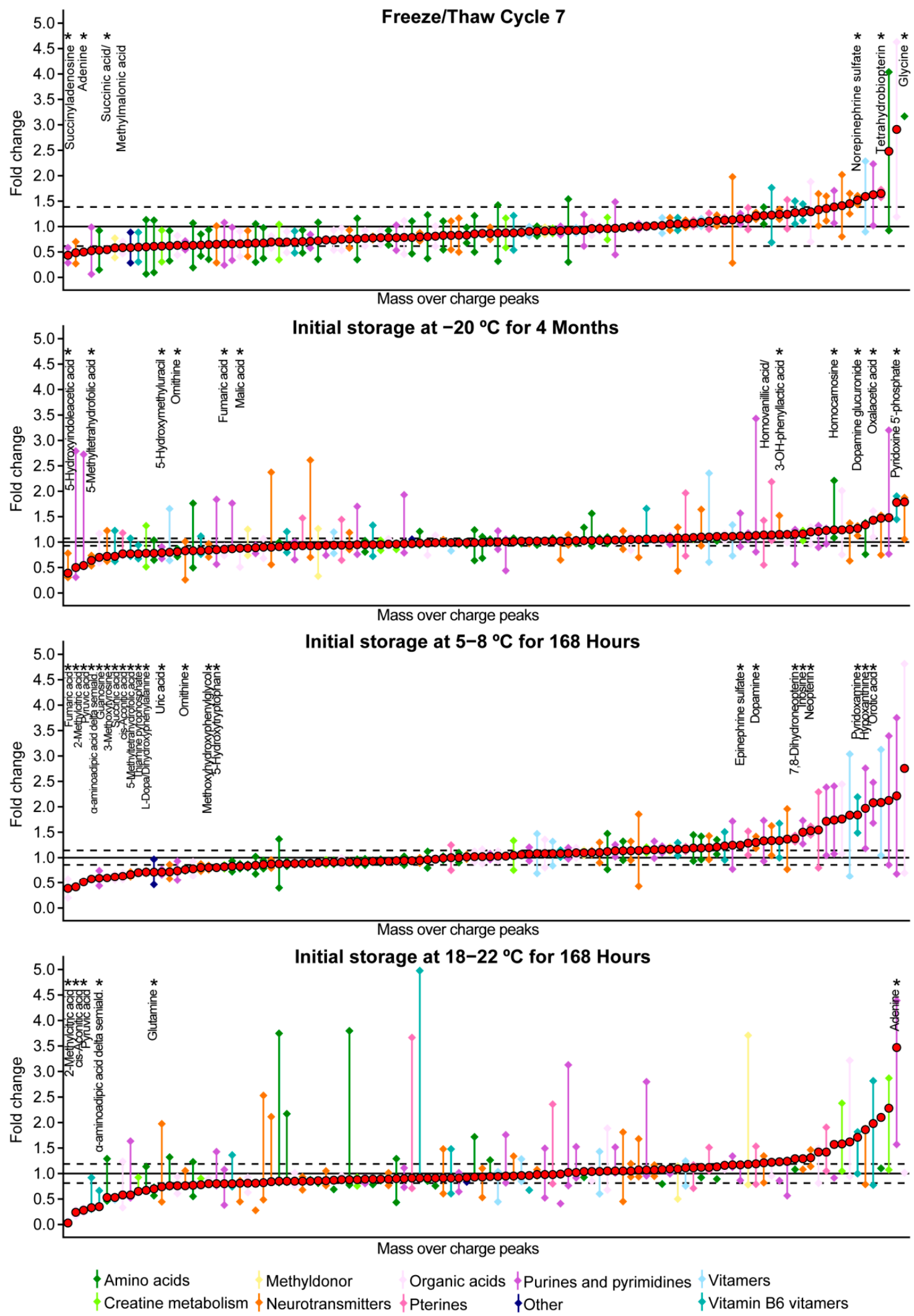

Figure 2. $m / z$ peaks corresponding to metabolites important for neurometabolic diagnostics that are possibly affected by the most extreme storage conditions compared to the reference sample, after correction for the analysis variability. The y-axis depicts the fold change of the most extreme storage condition compared to the reference sample; the x-axis depicts the $106 \mathrm{~m} / \mathrm{z}$ peaks corresponding to metabolites important for neurometabolic diagnostics (as listed in Table 2). Colored diamonds depict the upper and lower limit of the $95 \%$ confidence intervals; colored lines depict the range of the $95 \%$ confidence interval. Red filled circles depict the mean fold change. Colors depict the group of neurometabolic 
metabolites, as listed in the legend. The horizontal black line depicts fold change $=1$, horizontal dashed lines indicate the range of the analysis variability: fold change plus or minus the median absolute variation of the stable isotope-labeled compounds for the analysis series. Asterisks depict the metabolites for which the lower limit of $95 \%$ confidence interval was above the upper dashed line and metabolites for which the upper limit of the $95 \%$ confidence interval was below the lower dashed line. For these, metabolite names are indicated below the asterisks. These metabolites correspond to the metabolites illustrated in Figures S2-S5.

\section{Discussion}

We studied the effect of different storage conditions on the stability of small-molecule metabolites in CSF to aid a reliable interpretation of metabolomics results. We present two main findings. First, the majority of small-molecule metabolites seems to be relatively unaffected by multiple freeze/thaw cycles, as clustering analysis as well as repeated measures ANOVA comparing the different conditions and Kruskal-Wallis tests comparing the most extreme condition to the reference sample revealed no significant differences in metabolite intensities. Analysis of FCs revealed that adenine levels decreased more than $50 \%$ after more than four freeze/thaw cycles, and that glycine levels showed a five-fold increase after more than five freeze/thaw cycles (Figure S2). Two studies reported stable glycine concentrations in CSF after three freeze/thaw cycles, but these studies did not investigate the effects of more freeze/thaw cycles on glycine concentrations [7,8]. Altogether, we conclude that different numbers of freeze/thaw cycles in a single cohort are not expected to influence levels of small-molecule metabolites, but we suggest reticence in interpretation of potential biomarker concentrations in samples with more than four freeze/thaw cycles, especially with respect to adenine and glycine.

The second main finding is that the majority of small-molecule metabolites seems relatively unaffected by the time and temperature at which they were stored. For temperature stability analyses, clustering analysis as well as repeated measures ANOVA and Kruskal-Wallis tests revealed no significant differences, except for cis-aconitic acid when stored at $18-22{ }^{\circ} \mathrm{C}$. Analysis of FCs revealed that the levels of some metabolites, including 5-methyltetrahydrofolic acid, dopamine glucuronide, cis-aconitic acid, pyruvic acid, 2-methylcitric acid, $\alpha$-aminoadipic acid delta-semialdehyde, orotic acid, neopterin, glutamine and adenine might be affected by prolonged storage at $-20^{\circ} \mathrm{C}, 5-8{ }^{\circ} \mathrm{C}$, or $18-22{ }^{\circ} \mathrm{C}$ (Figure S3-S5). In another study, our group investigated the stability of 5-methyltetrahydrofolic acid in CSF samples and demonstrated decreased concentrations after a week of storage at room temperature, but, contrasting with our findings in this study, there were stable levels after storage for a week at $4{ }^{\circ} \mathrm{C}$ [9]. In the study previously performed by our group, storage at $-20^{\circ} \mathrm{C}$ was not included [9]. Another group found an increase of glutamine at $18-22^{\circ} \mathrm{C}$ [3], whereas we report a decrease of glutamine levels at this time point. Moreover, we did not observe decreased levels of citrate, nor increased levels of lactate, creatine, and creatinine. We observed decreased levels of pyruvic acid when stored at $18-22^{\circ} \mathrm{C}$, while Levine et al. reported stable levels of pyruvic avid [3]. Although Levine et al. used proton NMR analyses to detect potential differences, there were no other important differences in the study design that could explain these differences. Thus, the effects of prolonged storage at $-20{ }^{\circ} \mathrm{C}, 5-8{ }^{\circ} \mathrm{C}$, or $18-22{ }^{\circ} \mathrm{C}$ before final storage at $-80{ }^{\circ} \mathrm{C}$ are debatable. We suggest being cautious by including samples stored at $-20^{\circ} \mathrm{C}$ for more than two months (Figure S3), samples stored at $5-8{ }^{\circ} \mathrm{C}$ for more than $72 \mathrm{~h}$ (Figure S4), and samples stored at $18-22{ }^{\circ} \mathrm{C}$ for more than $8 \mathrm{~h}$ (Figure S5) in a cohort study for biomarker identification.

There are a few limitations to this study. First, while a wide range of pre-analytical conditions was studied, the sample size per condition was relatively small $(n=3)$, hampering the power of the analysis. For this reason, the relative stability of small-molecule metabolites in CSF might be overestimated, as biologically relevant changes in metabolite concentrations might not have reached statistical significance. Second, due to the exploratory nature of this study, surplus CSF samples were used that needed an extra thawing cycle for pooling. The reference sample thus underwent one 
freeze/thaw cycle instead of zero freeze/thaw cycles, hampering the interpretation of the effects of one freeze/thaw cycle on the concentrations of small-molecule metabolites.

Despite these limitations, an important strength of this study is that multiple statistical analyses were performed, including unsupervised clustering analysis, repeated measures ANOVA for comparing all storage conditions for each identified $m / z$ peak, Kruskal-Wallis tests for comparing the most extreme storage condition to the reference sample for each identified $\mathrm{m} / \mathrm{z}$ peak, and analysis of FCs with correction for the analysis variability to assess the more subtle differences in mass peak intensities. All these analyses pointed towards the same conclusion: concentrations of small-molecule metabolites are relatively unaffected by most pre-analytical storage conditions. Moreover, the nature of this study was untargeted, hypothesis-free, and broad, as the stability of more than $1800 \mathrm{~m} / \mathrm{z}$ peaks was tested.

\section{Materials and Methods}

\subsection{Sample Collection and Storage}

Surplus CSF samples from the Amsterdam Dementia Cohort (ADC) biobank (e.g., samples having incomplete clinical information for use in clinical validation studies), collected between 2000 and 2017, were used [10]. For the ADC biobank, CSF was collected by lumbar puncture in 10-mL polypropylene tubes (Sarstedt, Nümbrecht, Germany) and centrifuged at $1800-2100 \times g$ for $10 \mathrm{~min}$ at $4{ }^{\circ} \mathrm{C}$ within $2 \mathrm{~h}$, using a predefined procedure, according to international guidelines [11]. The CSF was divided into polypropylene tubes $\left(1.5\right.$ or $2.0 \mathrm{~mL}$; Sarstedt) in $500 \mu \mathrm{L}$ volumes and was stored at $-80^{\circ} \mathrm{C}[10,11]$.

The biobanking protocol of the ADC was approved by the review board of the Vrije Universiteit Medical Center of Amsterdam. All procedures followed were in accordance with the ethical standards of the institution and with the Helsinki Declaration of 1975, as revised in 2000. All subjects provided written consent.

Aliquots of $0.5 \mathrm{~mL}$ per sample from multiple CSF samples from three to six different individuals were merged into a CSF pool in 50-mL polypropylene tubes. In total, three CSF pools with different compositions were acquired. Aliquots of $500 \mu \mathrm{L}$ of these three CSF pools were stored in 1.5-mL polypropylene tubes with screw caps (Sarstedt). The aliquoted CSF pools were exposed to storage conditions as described in the standard operating procedure for sample stability [12] and as depicted in Figure 1, i.e., to assess the temperature stability of storage for up to four months at $-20^{\circ} \mathrm{C}$ and up to one week at either $5-8{ }^{\circ} \mathrm{C}$ or $18-22^{\circ} \mathrm{C}$ before final storage at $-80^{\circ} \mathrm{C}$; and to assess freeze/thaw stability exposure to up to seven freeze/thaw cycles (Figure 1). One reference aliquot of each pool was stored at $-80{ }^{\circ} \mathrm{C}$ directly at time point 0 . This sample had thus undergone one freeze/thaw cycle.

\subsection{Sample Preparation and Analysis}

Metabolomics analysis of CSF was performed as previously described by our group [5,6]. Samples were thawed to room temperature. Five microliters of the sample were added to $140 \mu \mathrm{L}$ of a working solution containing sILC, with fixed concentrations as previously described [6]. The solution was centrifuged for five minutes at $17,000 \times g$ and $105 \mu \mathrm{L}$ of supernatant was diluted with $45 \mu \mathrm{L} 0.3 \%$ formic acid (Emsure, Darmstadt, Germany). The solution was filtered using a methanol preconditioned 96-well filter plate (Acro prep, $0.2 \mu \mathrm{m}$ GHP, NTRL, $1 \mathrm{~mL}$ well; Pall Corporation, Ann Arbor, MI, USA) and a vacuum manifold. The sample filtrate was collected in a 96-well plate (Advion, Ithaca, NY, USA). Direct-infusion high-resolution mass spectrometry was performed using a TriVersa NanoMate system (Advion), controlled by Chipsoft software (version 8.3.3, Advion), that was mounted onto the interface of a Q-Exactive high-resolution mass spectrometer (Thermo Scientific ${ }^{\mathrm{TM}}$, Bremen, Germany), with a scan range of 70-600 m/z [6]. For each sample, technical triplicates were analyzed, infusing each sample three times into the mass spectrometer. Samples were analyzed in a randomized order. 


\subsection{Data Processing}

Data acquisition and processing were performed using a peak calling pipeline developed in the $\mathrm{R}$ programming language $[5,6]$. In this pipeline, mass peak identification and annotation was conducted by matching the $\mathrm{m} / \mathrm{z}$ value of the mass peak with a range of two parts per million to metabolite masses present in the Human Metabolome Database, version 3.6 [13]. According to the Metabolomics Standards Initiative, the level of certainty of metabolite annotation is 2, as we putatively annotate compounds based on the matched $m / z$ value of the mass peak [14]. Taking into account isomers and adduct ions, $\sim 60,000 \mathrm{~m} / z$ peaks could be annotated with one or more possible annotations [5]. Metabolite annotations without adduct ions in negative or positive mode $\left([\mathrm{M}-\mathrm{H}]^{-},[\mathrm{M}+\mathrm{H}]^{+}\right)$or with single adduct ions $[\mathrm{M}+\mathrm{Na}]^{+},[\mathrm{M}+\mathrm{K}]^{+}$and $[\mathrm{M}+\mathrm{Cl}]^{-}$were selected. For each sample, intensities of the five selected $\mathrm{m} / \mathrm{z}$ peaks were added together, resulting in one summed $\mathrm{m} / \mathrm{z}$ peak intensity per metabolite annotation: 6600 summed $\mathrm{m} / \mathrm{z}$ peaks in total, per sample. Endogenous metabolite annotations and metabolite annotations with unknown function were selected [5]. No normalization of mass peak intensities was performed. The R code is available online (https://github.com/UMCUGenetics/DIMS).

\subsection{Metabolite Groups}

To study metabolites known or expected to be important in neurometabolic diagnostics in CSF in detail (www.metagene.de) [15], $106 \mathrm{~m} / z$ peaks corresponding to neurometabolic metabolites were selected and grouped into different categories (Table 2).

\subsection{Data Analysis}

To assess which part of the identified variability is due to technical aspects of the analysis, the variability within the run was monitored by the addition of sILC to each sample during the sample analysis. For each SILC, the coefficient of variation was calculated over all samples in the run by standard deviation intensity/mean intensity. To assess the most extreme conditions of each series, these samples were compared to the reference sample by calculating the median absolute variation of the sILC as follows: (1) calculation of the fold change: intensity of the most extreme condition/intensity of the reference sample; (2) fold change - 1; (3) conversion to absolute numbers; (4) calculation of the median absolute variation: median of the absolute variation of the three CSF pools.

For the total number of $\mathrm{m} / \mathrm{z}$ peaks, as well as for the $106 \mathrm{~m} / \mathrm{z}$ peaks corresponding to neurometabolic metabolites, for each series a principal component analysis was performed to visualize whether there was any clustering of different freeze/thaw cycles or time points, or of the three CSF pools. In addition, for each series a repeated measures ANOVA for all storage conditions was performed for each of the $\mathrm{m} / \mathrm{z}$ peaks, as well as a non-parametric Kruskal-Wallis test comparing the most extreme condition to the reference sample for each of the $\mathrm{m} / z$ peaks. To control the familywise error rate in order to prevent any type I errors, all $p$-values were adjusted according to the Bonferroni method.

To assess which part of the identified variability is due to the different storage conditions, for each sample (except the reference sample), the FC was calculated as the intensity of the sample divided by the intensity of the reference sample. For each condition, the median absolute variation and $95 \% \mathrm{CI}$ of the three CSF pools were calculated. Next, to correct for variability that can solely be attributed to the analysis, metabolites were defined as possibly affected by the storage condition when the lower limit of $95 \%$ CI was above the range attributed to the analysis variability (calculated by: FC $1.000+$ median absolute variation of sILC), or when the upper limit of $95 \%$ CI was below the range attributed to the analysis variability (calculated by: FC 1.000 - median absolute variation of sILC). For each series, the median and $95 \% \mathrm{CI}$ were plotted for the most extreme condition. This was performed for the total number of $\mathrm{m} / \mathrm{z}$ peaks (data not shown), as well as for the $106 \mathrm{~m} / \mathrm{z}$ peaks corresponding to neurometabolic metabolites. To assess whether mass peak intensities were affected in specific groups of neurometabolic metabolites, metabolites were colored according to the metabolite group (Table 2). Data analysis was performed in the R programming language. 


\section{Conclusions}

In conclusion, we report that small-molecule metabolites in CSF are relatively unaffected by 1-3 freeze/thaw cycles, by storage at $-20{ }^{\circ} \mathrm{C}$ for up to two months, by storage at $5-8{ }^{\circ} \mathrm{C}$ up to $72 \mathrm{~h}$, or by storage at $18-22{ }^{\circ} \mathrm{C}$ up to $8 \mathrm{~h}$, suggesting that minor differences in pre-analytical storage conditions in multicenter or historical cohorts used for the identification of biomarkers will not affect the interpretation of potential biomarkers, and implying that these cohorts are suitable for biomarker studies. We recommend caution when including samples with more extreme pre-analytical storage conditions. In addition, prior to using a biomarker for clinical decision-making, we recommend that the effects of pre-analytical conditions on the concentrations of any newly identified potential biomarker are tested.

Supplementary Materials: The following are available online at http://www.mdpi.com/2218-1989/9/10/236/s1, Figure S1: Principal component analysis of the different storage conditions, Figure S2: Fold changes of $\mathrm{m} / \mathrm{z}$ peaks potentially affected by multiple freeze/thaw cycles, Figure S3: Fold changes of $m / z$ peaks potentially affected by prolonged storage at $-20^{\circ} \mathrm{C}$, Figure S4: Fold changes of $\mathrm{m} / \mathrm{z}$ peaks potentially affected by prolonged storage at $5-8{ }^{\circ} \mathrm{C}$, Figure S5: Fold changes of $m / z$ peaks potentially affected by prolonged storage at $18-22^{\circ} \mathrm{C}$.

Author Contributions: Conceptualization: H.A.H., E.A.J.W., C.E.T., N.M.V.-D., and J.J.M.J.; Formal analysis: J.G.; Funding acquisition: H.A.H. and J.J.M.J.; Investigation: H.A.H.; Methodology: H.A.H., E.A.J.W., C.E.T., N.M.V.-D., and J.J.M.J.; Resources: E.A.J.W., W.M.v.d.F., C.E.T., N.M.V.-D., and J.J.M.J.; Software: H.A.H.; Supervision: C.E.T., N.M.V.-D., and J.J.M.J.; Visualization: H.A.H. and E.A.J.W.; Writing—original draft: H.A.H.; Writing-review \& editing: E.A.J.W., J.G., W.M.v.d.F., C.E.T., N.M.V.-D., and J.J.M.J.

Funding: This work was supported by a personal Alexandre Suerman Stipend from the University Medical Centre Utrecht (H.A.H.) and by Metakids (2017-075) (J.J.M.J.).

Conflicts of Interest: The authors declare no conflict of interest. The funders had no role in the design of the study; in the collection, analyses, or interpretation of data; in the writing of the manuscript, or in the decision to publish the results.

\section{References}

1. Cameron, S.; Gillio-Meina, C.; Ranger, A.; Choong, K.; Fraser, D.D. Collection and analyses of cerebrospinal fluid for pediatric translational research. Pediatr. Neurol. 2019, 98, 3-17. [CrossRef] [PubMed]

2. Anesi, A.; Rondanelli, M.; d'Eril, G.M. Stability of neuroactive amino acids in cerebrospinal fluid under various conditions of processing and storage. Clin. Chem. 1998, 44, 2359-2360. [PubMed]

3. Levine, J.; Panchalingam, K.; McClure, R.J.; Gershon, S.; Pettegrew, J.W. Stability of CSF metabolites measured by proton NMR. J. Neural Transm. 2000, 107, 843-848. [CrossRef] [PubMed]

4. Rosenling, T.; Slim, C.L.; Christin, C.; Coulier, L.; Shi, S.; Stoop, M.P.; Bosman, J.; Suits, F.; Horvatovich, P.L.; Stockhofe-Zurwieden, N.; et al. The effect of preanalytical factors on stability of the proteome and selected metabolites in cerebrospinal fluid (CSF). J. Proteome Res. 2009, 8, 5511-5522. [CrossRef] [PubMed]

5. Haijes, H.A.; Willemsen, M.; van der Ham, M.; Gerrits, J.; Pras-Raves, M.L.; Prinsen, H.C.M.T.; van Hasselt, P.M.; de Sain-van der Velden, M.G.M.; Verhoeven-Duif, N.M.; Jans, J.J.M. Direct infusion based metabolomics identifies metabolic disease in patients' dried blood spots and plasma. Metabolites. 2019, 9, 12. [CrossRef] [PubMed]

6. Haijes, H.A.; van der Ham, M.; Gerrits, J.; van Hasselt, P.M.; Prinsen, H.C.M.T.; de Sain-van der Velden, M.G.M.; Verhoeven-Duif, N.M.; Jans, J.J.M. Direct-infusion based metabolomics unveils biochemical profiles of inborn errors of metabolism in cerebrospinal fluid. Mol. Genet. Metab. 2019, 127, 51-57. [CrossRef] [PubMed]

7. Fuchs, S.A.; de Sain-van der Velden, M.G.; de Barse, M.M.; Roeleveld, M.W.; Hendriks, M.; Dorland, L.; Klomp, L.W.; Berger, R.; de Koning, T.J. Two mass-spectrometric techniques for quantifying serine enantiomers and glycine in cerebrospinal fluid: Potential confounders and age-dependent ranges. Clin. Chem. 2008, 54, 1443-1450. [CrossRef] [PubMed]

8. Wilson, S.F.; James, C.A.; Zhu, X.; Davis, M.T.; Rose, M.J. Development of a method for the determination of glycine in human cerebrospinal fluid using pre-column derivatization and LC-MS/MS. J. Pharm. Biomed. Anal. 2011, 56, 315-323. [CrossRef] [PubMed] 
9. Willemse, E.A.J.; Vermeiren, Y.; Garcia-Ayllon, M.S.; Bridel, C.; De Deyn, P.P.; Engelborghs, S.; van der Flier, W.M.; Jansen, E.E.W.; Lopez-Font, I.B.; Mendes, V.; et al. Pre-analytical stability of novel cerebrospinal fluid biomarkers. Clin. Chim. Act. 2019, 497, 204-211. [CrossRef] [PubMed]

10. Van der Flier, W.M.; Pijnenburg, Y.A.; Prins, N.; Lemstra, A.W.; Bouwman, F.H.; Teunissen, C.E.; van Berckel, B.N.; Stam, C.J.; Barkhof, F.; Visser, P.J.; et al. Optimizing patient care and research: The Amsterdam Dementia Cohort. J. Alzheimers Dis. 2014, 41, 313-327. [CrossRef] [PubMed]

11. Del Campo, M.; Mollenhauer, B.; Bertolotto, A.; Engelborghs, S.; Hampel, H.; Simonsen, A.H.; Kapaki, E.; Kruse, N.; Le Bastard, N.; Lehmann, S.; et al. Recommendations to standardize pre-analytical confounding factors in Alzheimer's and Parkinson's disease cerebrospinal fluid biomarkers: An update. Biomark. Med. 2012, 6, 419-430. [CrossRef] [PubMed]

12. Andreasson, U.; Perret-Liaudet, A.; van Waalwijk van Doorn, L.J.; Blennow, K.; Chiasserini, D.; Engelborghs, S.; Fladby, T.; Genc, S.; Kruse, N.; Kuiperij, H.B.; et al. A practical guide to immunoassay method validation. Front. Neurol. 2015, 19, 179. [CrossRef] [PubMed]

13. Wishart, D.S.; Jewison, T.; Guo, A.C.; Wilson, M.; Knox, C.; Liu, Y.; Djoumbou, Y.; Mandal, R.; Aziat, F.; Dong, E.; et al. HMDB 3.0-The Human Metabolome Database in 2013. Nucleic Acids Res. 2013, 41, 801-807. [CrossRef] [PubMed]

14. Sumner, I.W.; Amberg, A.; Barrett, D.; Beale, M.H.; Beger, R.; Daykin, C.A.; Fan, F.W.; Fiehn, O.; Goodacre, R.; Griffin, J.L.; et al. Proposed minimum reporting standards for chemical analysis, Chemical Analysis Working Group (CAWG), Metabolomics Standards Initiative (MSI). Metabolomics 2007, 3, 211-221. [CrossRef] [PubMed]

15. Batllori, M.; Molero-Luis, M.; Casado, M.; Sierra, C.; Artuch, R.; Armazabal, A. Biochemical analyses of cerebrospinal fluid for the diagnosis of neurometabolic conditions. What can we expect? Semin. Pediatr. Neurol. 2016, 23, 273-284. [CrossRef] [PubMed]

(C) 2019 by the authors. Licensee MDPI, Basel, Switzerland. This article is an open access article distributed under the terms and conditions of the Creative Commons Attribution (CC BY) license (http://creativecommons.org/licenses/by/4.0/). 